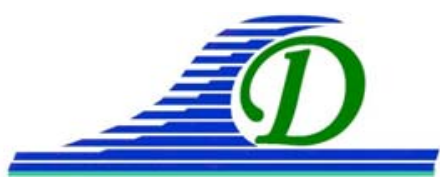
XIII ${ }^{\text {èmes }}$ Journées Nationales Génie Côtier - Génie Civil
Dunkerque, 2-4 juillet 2014

DOI:10.5150/jngcgc.2014.053 (c) Editions Paralia CFL

disponible en ligne - http://www.paralia.fr - available online

\title{
Modélisation numérique de l'impact des tempêtes sur une plage de poche partiellement aménagée
}

Denis MORICHON ${ }^{1,3}$, Iñaki DE SANTIAGO ${ }^{1,2}$, Stéphane ABADIE ${ }^{1,3}$

1. Université de Pau et des Pays de l'Adour, SIAME IVS, EA4581,

1 allée du parc Montaury, 64600 Anglet, France.

denis.morichon@univ-pau.fr

2. AZTI-tecnalia, Herrera Kaia, Portualdea z/g, E-20110 Pasaia, Gipuzkoa, Espagne.

3. Station Marine d'Anglet, Fédération MIRA FR2152, Observatoire Aquitain des

Sciences de l'Univers, Université de Pau et des Pays de l'Adour, Allée du parc

Montaury, 64600 Anglet, France.

\section{Résumé :}

La protection des zones côtières contre les vagues de tempête peut être assurée soit de façon naturelle, dans le cas de la présence d'un cordon dunaire, soit par des ouvrages maritimes sur les sites anthropisés. La plage de Zarautz, située au fond d'une baie, sur la côte basque espagnol, présente les deux types de protection sur un même linéaire. Des études récentes, basées sur l'analyse d'images vidéo et de levés topographiques réalisés avant et après une séquence de tempêtes, ont mis en évidence des variations morphologiques distinctes en fonction du niveau d'anthropisation de la plage. Afin de mieux comprendre la réponse de ce type de plage hybride à des événements fortement énergétiques, un modèle morphodynamique a été mis en place. Cette étude présente dans un premier temps la calibration du modèle en se basant sur un événement particulier ayant eu lieu en février 2012. Les résultats des simulations réalisées avec les paramètres optimaux, sont ensuite analysés en considérant différents secteurs de plage. L'étude présente ensuite les différentes phases d'évolution du profile de plage et du cordon dunaire au cours de la séquence de tempêtes.

Mots-clés : Impact des tempêtes, Erosion des dunes, Plage hybride, Xbeach, Morphodynamique, Ondes infra-gravitaires.

\section{Introduction}

Le littoral basque est caractérisé par une côte rocheuse. Par conséquent, les plages sableuses se situent majoritairement en fond de baie. Dans cette partie du golfe de Gascogne, les environnements côtiers sont soumis à de fortes contraintes sous l'action conjointe des vagues et des courants. Cette dernière décennie, la détérioration des falaises ou des digues de protection, qui constituent des remparts naturels ou artificiels, a été observée en de nombreux endroits. La protection et le maintien de plages d'agrément est aujourd'hui une préoccupation majeure du fait des enjeux socioéconomiques associés. 


\section{Thème 2 - Dynamique sédimentaire}

Différentes approches, plus ou moins complexes, permettent d'évaluer le risque associé aux tempêtes et d'estimer la résilience des ouvrages de protection ou d'un cordon dunaire. Une échelle d'impact de tempête (Storm Impact Scale) permet de prévoir la réponse d'un estran en fonction de sa topographie et d'un niveau de surcote estimé en fonction des conditions au large (SALLENGER, 2000). Cette approche relativement simple fournit un indicateur du risque potentiel de submersion ou de détérioration d'un cordon dunaire en cas de tempête. Toutefois, elle ne permet pas d'évaluer précisément le niveau d'endommagement des plages et des ouvrages de protection. L'érosion d'une plage lors d'une tempête est un phénomène rapide qui peut se traduire par des déplacements importants de sable et qui peut s'accompagner dans certains cas de la détérioration du cordon dunaire. Les modèles numériques tels que SBeach (LARSON \& KRAUS, 1989), Crosmor (VAN RIJN, 2009) et plus récemment XBeach (ROELVINK et al., 2009) ont été développés pour décrire l'évolution temporelle de la morphologie d'une plage lors d'une tempête. Ces modèles décrivent explicitement les flux sédimentaires et l'évolution du fond induits par les courants moyens générés par la transformation des vagues lors de leur propagation. L'érosion des dunes est prises en compte à partir d'une paramétrisation qui nécessite une calibration préalable du modèle. Cette étude porte sur l'application du modèle XBeach au site de la plage de Zarautz. Cette plage de poche présente la particularité de disposer d'un système de protection hybride constitué d'un cordon dunaire et d'un mur vertical. Le modèle Xbeach est utilisé afin d'étudier l'impact d'une séquence de tempêtes sur ce type de baie. La méthodologie appliquée pour déterminer les données nécessaires à la modélisation de l'événement de février 2012 est présentée dans la première partie. Ces données sont ensuite utilisées afin de calibrer le modèle. Dans la dernière section, l'évolution de la morphologie de la plage sur une période de 14 jours, comprenant 3 événements majeurs de tempête, est analysée à partir des résultats de la simulation numérique.

\section{Données disponibles}

\subsection{Site d'étude}

Le site de Zarautz est situé sur la côte basque espagnole. Il s'agit d'une plage de poche orientée selon la direction Nord-Nord Ouest. Les conditions climatiques présentent une forte saisonnalité associée à un régime de marée meso-tidale, avec un marnage maximum de $4.7 \mathrm{~m}$. La plage peut être divisée en deux sections (figure 1). Une partie "naturelle" située à l'Est qui présente un cordon dunaire de $10 \mathrm{~m}$ de haut et $600 \mathrm{~m}$ de long. A l'ouest du cordon dunaire, le trait de côte est entièrement endigué. Un mur vertical de $5 \mathrm{~m}$ de haut protège les habitations contre les risques de submersion. Il couvre une distance de $1.7 \mathrm{~km}$. La granulométrie varie le long de la plage. On trouve les sédiments les plus fins dans la partie Ouest aménagée $\left(D_{50}=0.2 \mathrm{~mm}\right)$, et les sédiments plus grossiers sur la partie naturelle, à l'Est de la plage $\left(D_{50}=0.45 \mathrm{~mm}\right)$. 


\section{XIII ${ }^{\text {èmes }}$ Journées Nationales Génie Côtier - Génie Civil \\ Dunkerque, 2-4 juillet 2014}

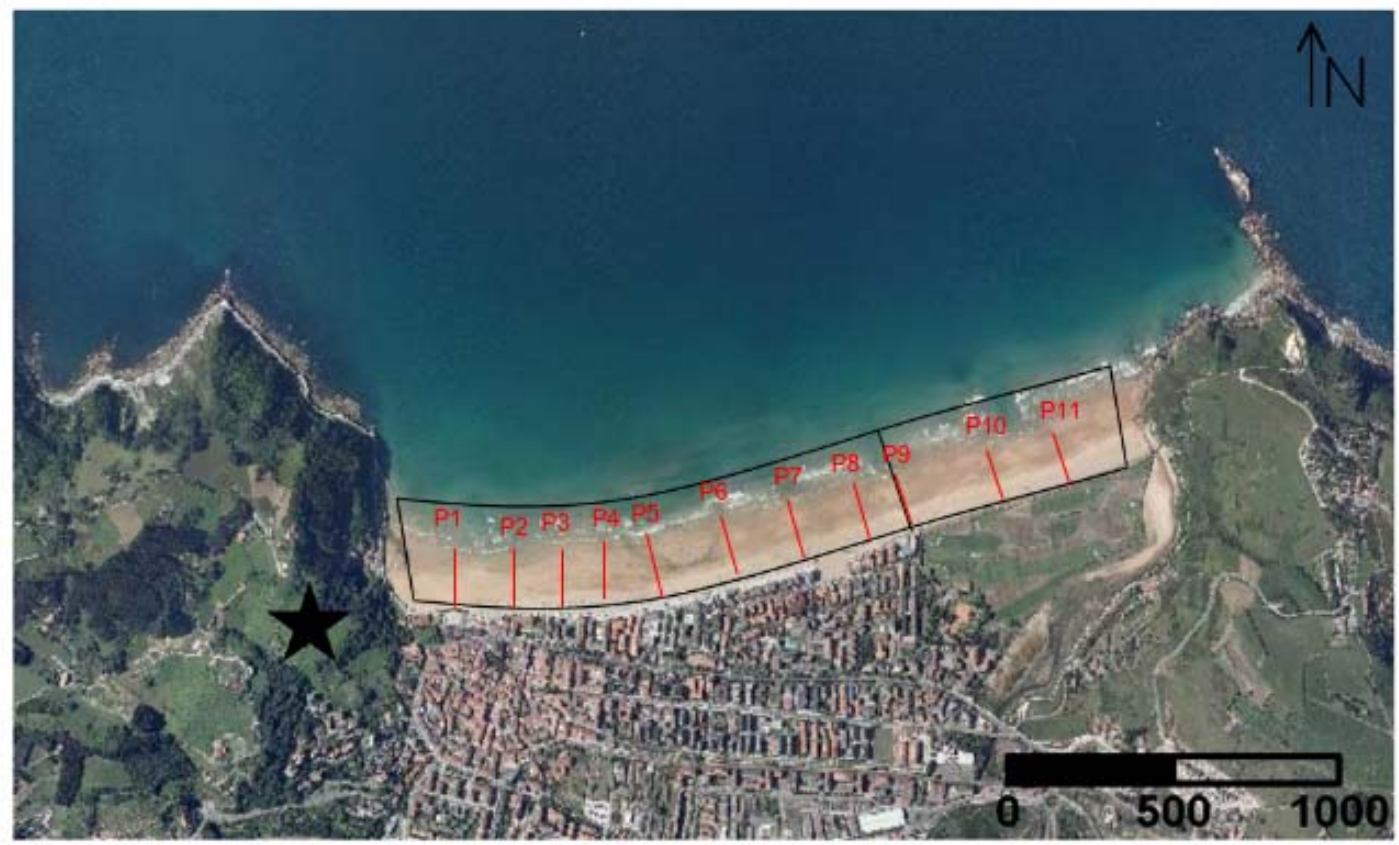

Figure 1. Présentation du site d'étude et position des profils le long de la partie aménagée (P1-P9) et le long du cordon dunaire (P10 - P11)

D'un point de vue morphologique, le site de Zarautz est dominé par un système de double barres. Lors d'événements faiblement énergétiques suffisamment longs, on observe une migration de la barre externe vers la côte qui peut aller jusqu'à sa connexion avec la barre interne. Plusieurs emplacements préférentiels des courants d'arrachement ont été identifiés le long de la plage, ce qui semble indiquer que les effets des promontoires rocheux ne sont pas localisés mais s'étendent à la circulation de l'ensemble de la baie (DE SANTIAGO et al., 2013).

\subsection{Données de vagues}

Les conditions d'agitation au large du site de Zarautz sont obtenues à partir d'une bouée de houle directionnelle, ancrée à $600 \mathrm{~m}$ de fond au large de Bilbao. Ces données ont été propagées pour rendre compte de la transformation du régime de vague lors de sa propagation du point de mesure jusqu'au site de Zarautz. Afin de réduire les temps de calcul, un modèle "simple" de propagation a été développé. La première étape a consisté à analyser le climat de houle obtenu à partir d'une base de données couvrant une période de neuf ans de mesures. Cette analyse a permis de mettre en évidence que l'énergie des spectres de vague mesurés au large de Bilbao est concentrée autour de 270 à 360 degrés, avec des fréquences situées entre $0.16 \mathrm{~Hz}$ et $0.0625 \mathrm{~Hz}$. Le spectre de vague utilisé pour la simulation a été filtré pour ne conserver que cette gamme de directions et de fréquences. Le spectre ainsi filtré a été divisé en plusieurs bandes qui ont ensuite été 
propagées avec le modèle REF-DIF en prenant une valeur de hauteur de vague nominale $H$ de $1 \mathrm{~m}$.
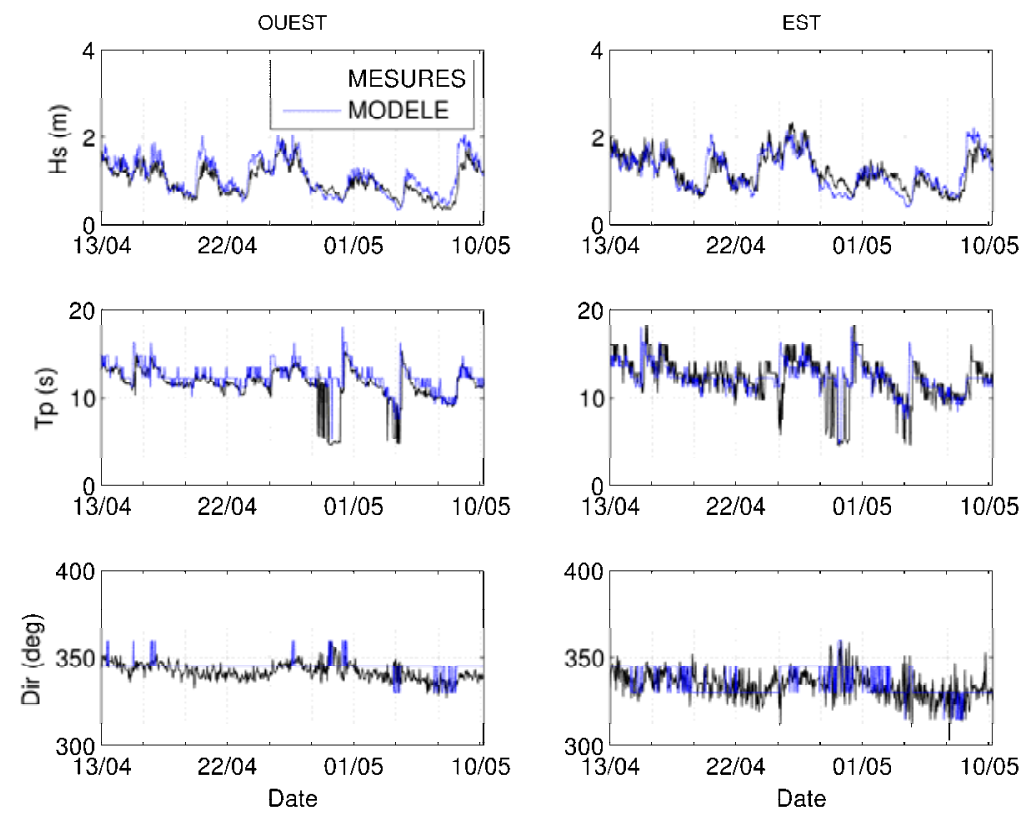

Figure 2. Comparaison entre les caractéristiques de vagues calculées et mesurées en deux points au large de Zarautz en 2011.

On obtient alors une matrice de transformation du spectre de vague. Les caractéristiques des vagues telles que la hauteur de vague significative $H_{s}$, la période pic $T_{p}$ ainsi que la direction $D_{p}$ au large de Zarautz sont alors obtenues en appliquant cette matrice de transformation au spectre de vague mesuré par la bouée de Bilbao.

Le modèle a été validé avec des données de houles mesurées au large de la baie de Zarautz avec deux ADCP mouillés à $18 \mathrm{~m}$ de profondeur, respectivement à $\mathrm{l}$ 'Est et à l'Ouest de la baie, pendant un mois. La figure 2 présente une comparaison entre les caractéristiques du spectre de vague mesurées et les valeurs calculées. Le modèle reproduit correctement les mesures. Il permet de rendre compte de la variabilité des conditions d'agitation au large de la baie. On remarque notamment un plus grand étalement directionnel à l'Est de la baie. Les différences les plus importantes concernent la période pic. Autour du 1er mai, des périodes de l'ordre de $5 \mathrm{~s}$ ont été mesurées. Elles correspondent à une mer de vent probablement générée localement. Notre approche par définition ne permet pas de rendre compte de ces états de mer.

\subsection{Données topographiques et bathymétriques}

Au cours de la période d'étude, qui s'étend du $1^{\text {er }}$ au 14 février 2012, deux campagnes de levés topographiques ont été réalisées, au début et à la fin de la période, afin de mesurer 


\section{XIII ${ }^{\text {èmes }}$ Journées Nationales Génie Côtier - Génie Civil \\ Dunkerque, 2-4 juillet 2014}

la réponse de la plage à une séquence d'événements de tempête. Lors de ces deux campagnes de mesure, 11 profils couvrant les différents secteurs de la plage ont été mesurés au GPS Trimble RTK-GNSS (Real Time Kinematic Global Navigation

Satellite System). Les profils sont distants de $200 \mathrm{~m}$ et l'élévation de la plage le long d'un profil est mesurée tous les $3 \mathrm{~m}$. Les profils s'étendent de la base du mur de protection, et du sommet du cordon dunaire, jusqu'au niveau des plus basses mers (figure 1). Les conditions climatiques (figure 4) n'ont pas permis de réaliser de mesures bathymétriques au cours de la période d'étude. Par conséquent, ces données ont été estimées en appliquant le modèle d'assimilation de données Beach Wizard (VAN DONGEREN et al., 2008). Le modèle consiste à appliquer un algorithme de corrélation entre des cartes de dissipation d'énergie issues d'images vidéo ortho-rectifiées, et des cartes issues de résultats de simulations réalisées avec le module de vague du code XBeach. Les images sont obtenues à partir d'une station vidéo installée à l'ouest de la baie à 90 m d'altitude (DE SANTIAGO et al., 2013).

\section{Modèle numérique}

\subsection{Xbeach}

Le code open-source XBeach (ROELVINK et al., 2009) permet de simuler l'impact des événements climatiques extrêmes sur l'évolution morphologique des plages sableuses en mettant l'accent sur la réponse du haut de plage. Il s'agit d'un modèle morphodynamique simulant les vagues avec une approche à phase moyennée tandis que la phase des ondes infragravitaires est résolue. Les courants moyens induits sont ensuite utilisés pour calculer le transport sédimentaire et l'évolution du fond associée. Ce modèle fait intervenir de nombreux paramètres libres et nécessite donc une calibration pour chaque site d'étude.

\subsection{Calibration du modèle}

Afin de limiter le nombre de tests, une étude préliminaire a permis de déterminer les paramètres libres qui influencent de façon significative les résultats du modèle. Un des paramètres les plus importants concerne le paramètre qui précise le choix de la formule de transport sédimentaire. Les simulations ont été réalisées en considérant l'ensemble de la plage. Un maillage irrégulier, raffiné à partir de la zone de déferlement, a été utilisé pour limiter les temps de calcul. Les meilleurs résultats ont été obtenus avec la formule de Van Rijn- Reniers Van Thiel (VAN THIEL DE VRIES, 2010). Les résultats sont également sensibles aux valeurs des paramètres facua et wetsl. Le paramètre facua permet de prendre en compte l'influence de l'asymétrie des vagues sur la direction du transport sédimentaire. Une valeur de facua $=0$ implique un transport dirigé exclusivement vers le large. Dans notre étude, la durée de l'événement utilisé pour la 


\section{Thème 2 - Dynamique sédimentaire}

calibration implique qu'entre les tempêtes une partie du transport sédimentaire puisse se faire vers la côte. Par conséquent, plusieurs valeurs de facua $>0$ ont été testées.
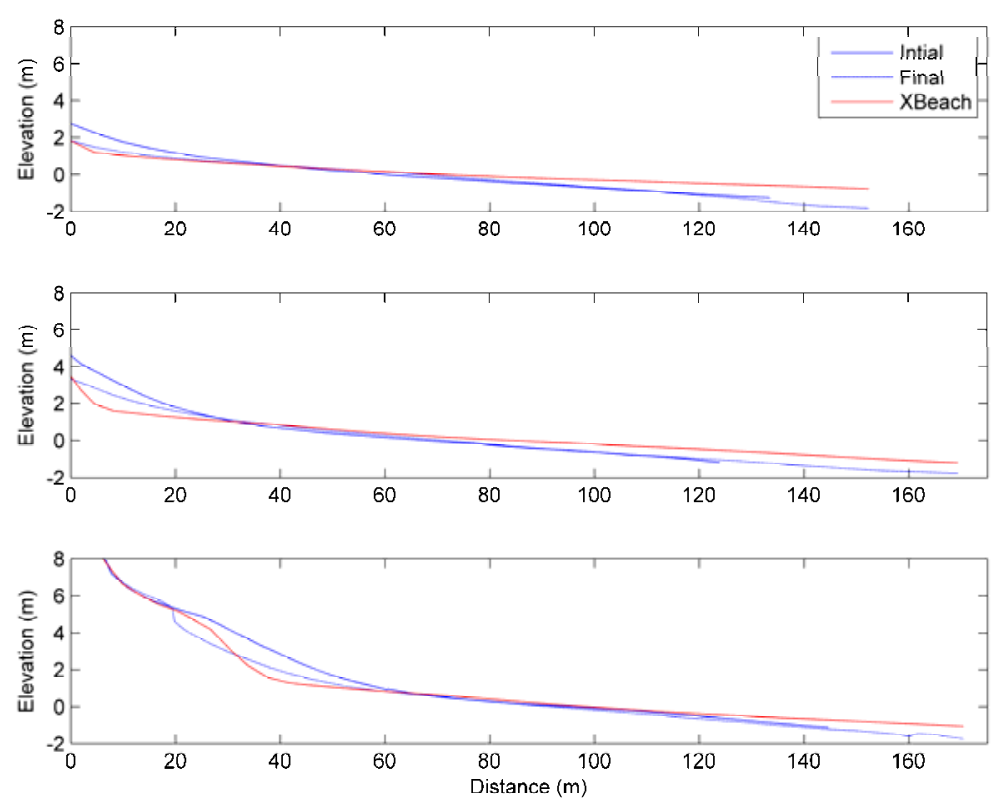

Figure 3. Résultats de la calibration du modèle pour 3 profiles avec de haut en bas les profils $\mathrm{P3}(\mathrm{RMS}=0.44 \mathrm{~m}), \mathrm{P9}(\mathrm{RMS}=0.44)$ et $\mathrm{P} 11(\mathrm{RMS}=0.36)$

La réponse d'un cordon dunaire à une tempête n'est pas résolue de manière explicite dans Xbeach. Un algorithme d'avalanche (ROELVINK et al., 2009) permet d'éroder la dune en fonction de la valeur de la pente locale par rapport à une valeur spécifiée par le paramètre wetsl, qui caractérise la pente critique de plage mouillée.

La figure 3 présente les résultats du modèle obtenus avec les paramètres optimaux ( facua $=0.2$, wetsl $=0.3$ ) qui correspondent à une erreur RMS minimale de $0.5 \mathrm{~m}$, erreur calculée en considérant l'ensemble de la plage. On remarque que le modèle reproduit correctement l'érosion du haut de plage dans la partie Est (P3) et Ouest (P11) de la plage. Dans la zone de transition (P9), il surestime le volume de sable érodé. Le recul du pied de dune (P11), de l'ordre de $4.5 \mathrm{~m}$, est comparable aux mesures mais la pente diffère.

\section{Analyse des résultats}

Au cours de la période d'étude, la plage de Zarautz a subi 3 événements de tempête de forte intensité $\left(H_{s, \max }=6 \mathrm{~m}\right)$, combiné à des marnages importants pour le dernier événement (figure 4). L'étude de l'évolution des profils P3, P9 et P11, calculés avec le modèle Xbeach, après chaque tempête montre des comportements différents en fonction de la position du profil le long de la plage. 


\section{XIII ${ }^{\text {èmes }}$ Journées Nationales Génie Côtier - Génie Civil \\ Dunkerque, 2-4 juillet 2014}
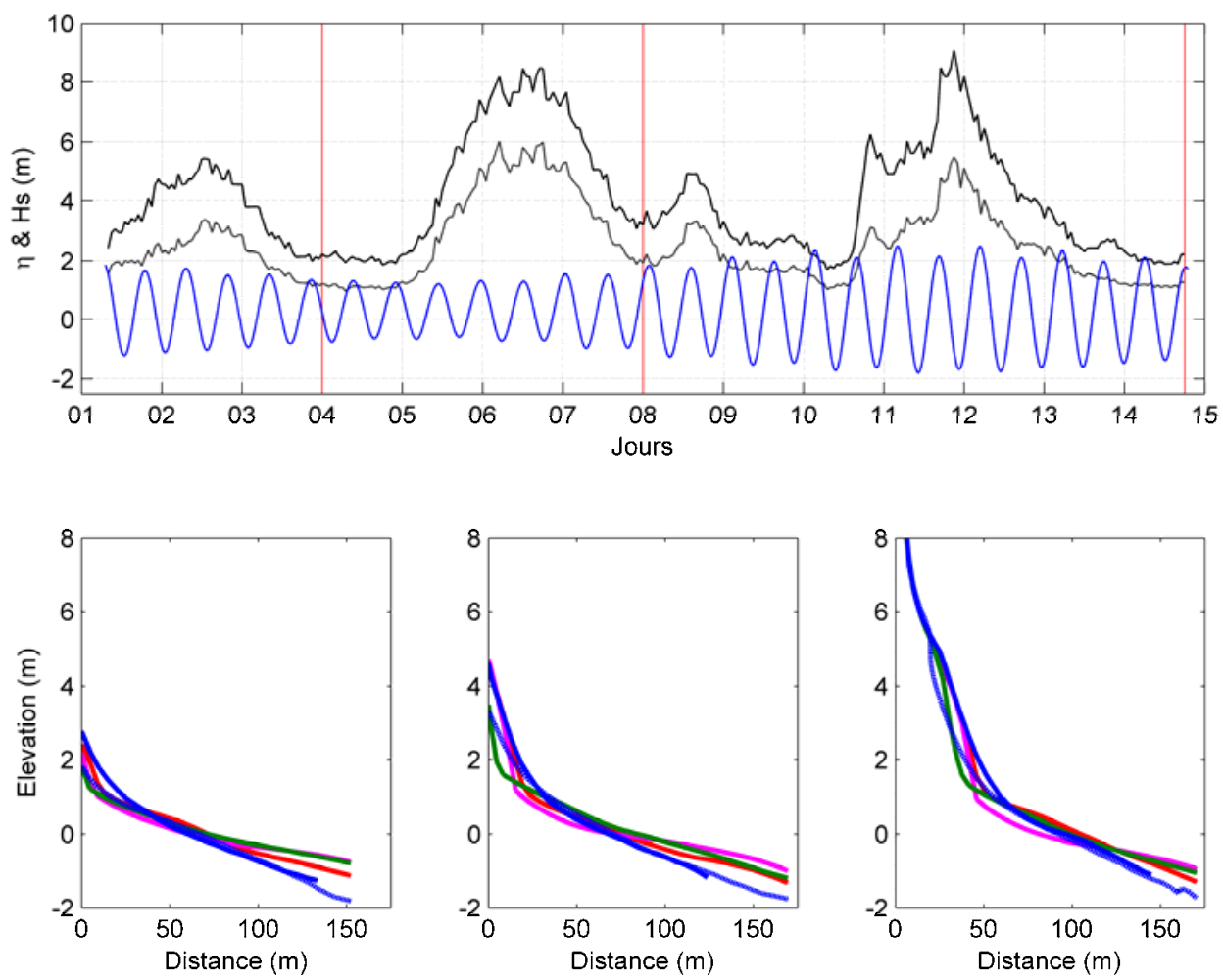

Figure 4. Conditions d'agitation (en noir bouée de Bilbao, en gris calculé au large de Zarautz) et évolution des profils $\mathrm{P} 3, \mathrm{P} 9, \mathrm{P} 11$ (de gauche à droite) après chaque tempête Tn (rouge T1, violet T2, vert T3), les profils en bleu correspondent aux mesures (profil initial en trait plein et profil final en pointillé)

L'essentiel de l'évolution le long du profil P3, situé dans la partie aménagée de la plage, semble se produire au cours de la première tempête puis tend vers une forme d'équilibre. Au niveau de la zone de transition, le profil P9 est actif au cours des 3 tempêtes. Sur cette partie de la plage, le modèle surestime le volume de sable érodé. Au niveau du cordon dunaire, on constate un faible impact de la première tempête certainement du fait d'un niveau d'eau moyen relativement faible qui a limité l'érosion du pied de dune. Au cours de la seconde tempête, beaucoup plus énergétique, on constate une forte érosion de l'estran sans endommagement majeur de la dune. Le recul important du trait de côte, observé en comparant les profils mesurés au début et à la fin de la période d'étude, se produit au cours de la troisième tempête qui coïncide avec un marnage élevé.

\section{Conclusion}

Dans cette étude, nous avons appliqué le modèle XBeach afin de simuler la réponse de la plage de Zarautz a des événements fortement énergétiques. L'analyse des résultats numériques montre des comportements différents en fonction des secteurs de plage, ce 
qui est conforme avec les précédentes études (DE SANTIAGO et al., 2013). Le modèle permet d'étudier l'évolution de la plage au cours d'une séquence de plusieurs tempêtes et d'identifier les conditions pour lesquelles le cordon dunaire est le plus vulnérable. L'estimation de la bathymétrie à partir de BeachWizard peut expliquer les différences observées entre la simulation et les mesures. Des analyses complémentaires sont nécessaires pour comprendre l'influence des ouvrages de protection sur l'hydrodynamique et les mouvements sédimentaires, en particulier au niveau de la zone de transition.

\section{Remerciements}

Les auteurs remercient les Ports d’États Espagnols (Puertos del Estado, Ministère espagnol) pour la mise à disposition des données de houle issues du houlographe de Bilbao.

\section{Références bibliographiques}

DE SANTIAGO I., MORICHON D., ABADIE S., LIRIA P., EPELDE I. (2013). Effect of winter storms on a partially engineered embayed beach: the case of Zarautz beach (north of Spain). Proceeding of Coastal Dynamics 2013, Arcachon, France, pp 487-497. LARSON M., KRAUS N. (1989). SBEACH: Numerical Model for Simulating Storminduced Beach Change. Report 1, Empircal Foundation and Model Development. Technical Report CERC 89-9. Coastal Engineering Research Center.

ROELVINK D., RENIERS A., VAN DONGEREN A., VAN THIEL DE VRIES J., McCALL R., LESCINSKI J. (2009). Modelling storm impacts on beaches, dunes and barrier islands. Coastal Engineering, Vol. 56, pp 1133-1152. http://dx.doi.org/10.1016/j.coastaleng.2009.08.006

SALLENGER A.H.J. (2000). Storm impact scale for barrier islands. Journal of Coastal Research, Vol. 16, pp 890-895.

VAN DONGEREN A., PLANT N., COHEN A., ROELVINK D., HALLER M., CATALÀN P. (2008). Beach Wizard: Nearshore bathymetry estimation through assimilation of model computations and remote observations. Coastal Engineering, Vol. 55, pp 1016-1027. http://dx.doi.org/10.1016/j.coastaleng.2008.04.011

VAN RIJN L.C. (2009). Prediction of dune erosion due to storms. Coastal Engineering, Vol. 56, pp 441-457. http://dx.doi.org/10.1016/j.coastaleng.2008.10.006

VAN THIEL DE VRIES J.S.M. (2010). Dune erosion during storm surges. PhD Thesis. Delft University of Technology, Delft. 